Linked Article: Yiu et al. Br J Dermatol 2020; [bjd.18981]

\title{
Biologics for psoriasis: what does drug survival tell us?
}

Drug survival is defined as the duration of treatment from initiation to discontinuation and is utilized as a proxy for effectiveness, incorporating tolerability, safety, and patient and physician preferences. ${ }^{1,2}$ Drug survival studies aim to describe the real-world performance of biologic therapies and address the lack of external validity provided by randomized controlled trials (RCTs).

In this edition of the $B J D$, Yiu et al. ${ }^{3}$ report the findings of a drug survival analysis utilizing data from the British Association of Dermatologists Biologics and Immunomodulators Register (BADBIR) between 2007 and 2019. BADBIR is one of the largest dermatology databases in the world, with prospectively recorded data from 164 participating dermatology centres across the UK and Republic of Ireland. ${ }^{3}$ Discontinuation was defined as ceasing treatment for more than 90 days, and survival was analysed according to reason for discontinuation (ineffectiveness, adverse effects, other). Multiple clinical and demographic factors were included as potential confounders in the survival analysis model. In total 9652 patients were included: 5543 on adalimumab, 3118 on ustekinumab and 991 on secukinumab. Secukinumab and ustekinumab had similarly high and sustained survival after 2 years for each discontinuation category and overall. Adalimumab had the lowest survival function at all timepoints for all categories. Survival stratified by adverse effects was no different between ustekinumab and secukinumab, and slightly worse for adalimumab. The adjusted hazard ratios of discontinuing adalimumab and secukinumab compared with ustekinumab due to ineffectiveness were 2.11 (95\% confidence interval 1.76-2.54) and 0.67 (95\% confidence interval $0.4-1.11$ ), respectively.

The results are of particular interest for a number of reasons. Secukinumab has shown poorer drug survival than expected from RCT outcomes. ${ }^{4-7}$ In addition, despite having better efficacy than ustekinumab in clinical trials, ${ }^{8}$ it has compared unfavourably in drug survival studies. ${ }^{9}$

These results were likely distorted by low rates of biologic-naive patients (18-40\%) and small This is the author manuscript accepted for publication and has undergone full peer review but has not been through the copyediting, typesetting, pagination and proofreading process, which may lead to differences between this version and the Version of Record. Please cite this article as doi: 10.1111/BJD.19189

This article is protected by copyright. All rights reserved 
patient numbers, ${ }^{6,7,9}$ with those on secukinumab possibly representing a particularly hard-totreat group. In contrast, biologic-naive patients constituted $72.9 \%$ of the BADBIR secukinumab cohort of 991 patients, the largest sample size to date. Not only did secukinumab and ustekinumab have similar adjusted survival for effectiveness, both agents were equally susceptible to earlier discontinuation in the biologic-experienced group compared with the biologic naive.

Adalimumab survival was not significantly affected by previous biologic exposure, and all three agents had similar drug survival in the biologic-experienced group by year 2. Psoriatic arthritis predicted cessation of ustekinumab and persistence of adalimumab and secukinumab; however, adalimumab still had the poorest drug survival in patients with or without psoriatic arthritis. Strikingly, other than psoriatic arthritis and previous biologic experience, the many other clinical factors included in the adjusted analysis were not differentially predictive of drug survival between the three agents. Of note, the palmoplantar psoriasis subtype was not predictive of discontinuing, although it has been established as a risk factor for poorer response to biologic therapy. ${ }^{10}$ The authors propose that determinants of survival may reside instead in pharmacokinetic, genomic, transcription and adherence factors rather than clinical variables.

Validity of the results is supported by methodological strengths limiting bias and confounding, in particular the sample size and coverage, the level of precision as indicated by the confidence intervals, the detailed data on reasons for discontinuing, and the inclusion of multiple covariates as potential confounders. Sensitivity analysis restricting data to when all three biologics were available in BADBIR (from 2013), with no change in the direction of the results, also improves the certainty. The limitations are discussed in full by the authors, including the lack of randomization and residual confounding and missing data, in particular Dermatology Life Quality Index scores. Not discussed is the lack of inclusion of employment status as a covariate, which is available from BADBIR and has been shown to influence efficacy; ${ }^{10}$ it is therefore a likely confounder.

The authors conclude that the results support the British Association of Dermatologists' recommendation of secukinumab as a first-line biologic therapy along with ustekinumab and adalimumab; however, this may be altered as registry data accumulate on the newer antiinterleukin-17 and anti-interleukin-23 agents. ${ }^{11}$

How should we interpret drug survival for use in clinical practice? Drug survival analysis as a proxy for effectiveness and safety has been criticized as possibly misleading given the range of potential reasons for ceasing a drug, such as frequency of dosing, a myriad of prescriber 
and patient factors, and discontinuing due to both good (remission) and bad outcomes. ${ }^{12}$ Mason et al. also established that drug survival (even restricted to those ceasing due to ineffectiveness) does not necessarily improve with Psoriasis Area and Severity Index score reduction. ${ }^{13}$ Furthermore, there is high heterogeneity in drug survival study methodology and reporting, ${ }^{14}$ with minimal adherence to proposed guidelines on study design, ${ }^{2}$ therefore limiting their utility, comparability and value to meta-analyses. However, even adhering to a gold-standard design, drug survival remains a composite measure that suffers from a lack of data on health-related quality of life and other measurable covariates such as adherence and socioeconomic status. These limitations notwithstanding, it is still a useful adjunct to RCTbased efficacy data for clinical decision making.

In conclusion, Yiu and colleagues present a robust survival analysis and, as such, near definitive evidenee that a wide range of clinical factors do not differentiate drug survival between adalimumab, ustekinumab and secukinumab, and that secukinumab and ustekinumab perform similarly according to this metric over 2 years. Journals should expect drug survival studies to comply with agreed methodology and reporting standards to allow valid comparisons and meta-analyses.

Acknowledgments: I thank Associate Professor Chris Baker for his critical review of this commentary

E. Veysey

Dermatology, St Vincent's Hospital, Fitzroy, Melbourne, VIC, Australia

Email: ecveysey@gmail.com

https://orcid.org/0000-0002-1782-9945 (URL checked and working)

Conflicts of interest: the author declares they have no conflicts of interest.

\section{References}

1 Navarini AA, French LE. Survival of second-line biologics in psoriasis: the British BADBIR Registry Data informs daily practice. J Invest Dermatol 2018; 138:726-8. 2 van den Reek JMPA, Kievit W, Gniadecki R et al. Drug survival studies in dermatology: principles, purposes, and pitfalls. J Invest Dermatol 2015; 135:1-5. 
3 Yiu ZZN, Mason KJ, Hampton PJ et al. Drug survival of adalimumab, ustekinumab and secukinumab in patients with psoriasis: a prospective cohort study from the British Association of Dermatologists Biologics and Immunomodulators Register (BADBIR). Br J Dermatol 2020; in press.

4 Georgakopoulos JR, Ighani A, Phung M, Yeung J. Drug survival of secukinumab in realworld plaque psoriasis patients: a 52-week, multicenter, retrospective study. J Am Acad Dermatol 2018; 78:1019-20.

5 Lee EB, Amin M, Egeberg A, Wu JJ. Drug survival of secukinumab for psoriasis in a realworld setting. J Dermatolog Treat 2019; 30:150-1.

6 van den Reek JMPA, van Vugt LJ, van Doorn MBA et al. Initial results of secukinumab drug survival in patients with psoriasis: a multicentre daily practice cohort study. Acta Derm Venereol 2018; 98:648-54.

7 Egeberg A, Bryld LE, Skov L. Drug survival of secukinumab and ixekizumab for moderateto-severe plaque psoriasis. $J$ Am Acad Dermatol 2020; in press.

8 Blauvelt A, Reich K, Tsai T-F et al. Secukinumab is superior to ustekinumab in clearing skin of subjects with moderate-to-severe plaque psoriasis up to 1 year: results from the CLEAR study. J Am Acad Dermatol 2017; 76:60-9.

9 Egeberg A, Ottosen MB, Gniadecki R et al. Safety, efficacy and drug survival of biologics and biosimilars for moderate-to-severe plaque psoriasis. Br J Dermatol 2018; 178:509-19. 10 Warren RB, Marsden A, Tomenson B et al. Identifying demographic, social and clinical predictors of biologic therapy effectiveness in psoriasis: a multicentre longitudinal cohort study. Br J Dermatol 2019; 180:1069-76.

11 Smith CH, Jabbar-Lopez ZK, Yiu ZZ et al. British Association of Dermatologists guidelines for biologic therapy for psoriasis 2017. Br J Dermatol 2017; 177:628-36. 12 Dávila-Seijo P, García-Doval I. Drug survival analysis is not a good method for assessing the safety or effectiveness of systemic therapies in psoriasis. Actas Dermosifiliogr 2017;

108:3-5.

13 Mason KJ, Barker JNWN, Smith CH et al. Comparison of drug discontinuation, effectiveness, and safety between clinical trial eligible and ineligible patients in BADBIR. JAMA Dermatol 2018; 154:581-8.

14 Costanzo A, Malara G, Pelucchi C et al. Effectiveness end points in real-world studies on biological therapies in psoriasis: systematic review with focus on drug survival. Dermatology (Basel) 2018; 234:1-12. 


\section{University Library}

\section{- M M I N E R VA A gateway to Melbourne's research publications}

Minerva Access is the Institutional Repository of The University of Melbourne

Author/s:

Veysey, E

Title:

Biologics for psoriasis: what does drug survival tell us?

Date:

2020-06-10

Citation:

Veysey, E. (2020). Biologics for psoriasis: what does drug survival tell us?. BRITISH

JOURNAL OF DERMATOLOGY, 183 (2), pp.204-205. https://doi.org/10.1111/bjd.19189.

Persistent Link:

http://hdl.handle.net/11343/275887 